\title{
Cartas ao Editor
}

\section{A equação de transporte de Boltzmann e sua importância para a física dos reatores nucleares}

De parabéns está a SBF, ao editar na Revista Brasileira do Ensino de Física, uma edição em tributo a um dos pilares da Física, Ludwig Boltzmann, por ocasião do centenário de sua morte. Porém a contribuição de Boltzmann vai além da fundação da mecânica estatística, sendo que a sua famosa equação descrevendo a distribuição esperada de partículas, no espaço de fases, $n(\mathbf{r}, \mathbf{v}, t)$, i.e.

$$
\frac{\partial n}{\partial t}+\mathbf{v} \cdot \nabla_{\mathbf{r}} n+\frac{\mathbf{F}}{m} \cdot \nabla_{\mathbf{v}} n=\left(\frac{d n}{d t}\right)_{\text {colisões }},
$$

com $\mathbf{F}$ a força externa e o termo $(d n / d t)$ colisões representando a variação total no número esperado de partículas devido a colisões é o modelo básico para a descrição de transporte de partículas, quer para os chamados processos de caminho aleatório ("randon walk", ou auto-difusão), ou de processos de transporte coletivo, conforme ilustrado na Fig. 1.

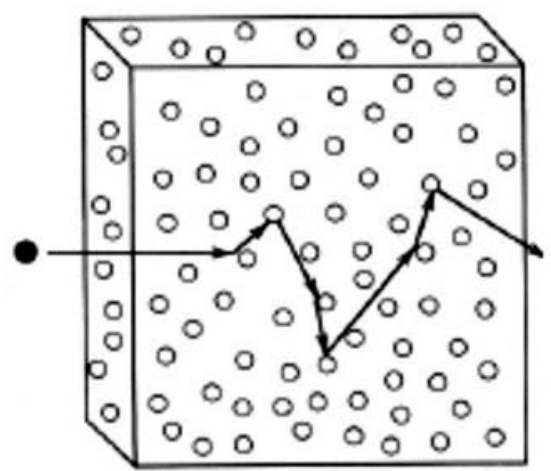

Processo de

auto-difusão ou "random walk"

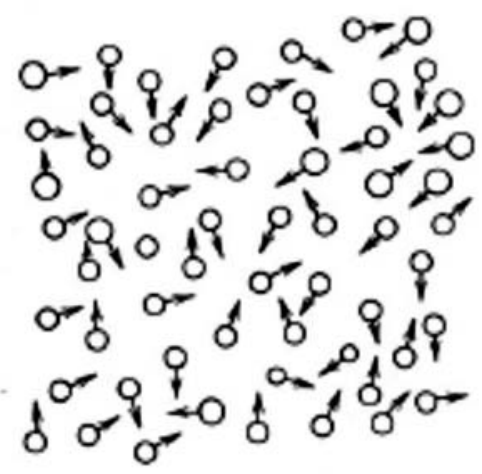

Processo coletivo

Figura 1 - Ilustração dos processos de transporte.
No caso de partículas sem carga (e.g. nêutrons e fótons), o processo de transporte é de auto-difusão,e com base nas hipóteses físicas, tais como a densidade das partículas muito menor do que a densidade das partículas do meio em que ocorre o transporte, a não interação entre partículas, e a ausência de forças externas, "heuristicamente" pode-se mostrar que

$$
\begin{array}{r}
\left(\frac{d n}{d t}\right)_{\text {colisões }}=-v \Sigma_{t}(\mathbf{r}, \mathbf{v}) n(\mathbf{r}, \mathbf{v}, t)+ \\
\int d^{3} v^{\prime} v^{\prime} \Sigma\left(\mathbf{r}, \mathbf{v}^{\prime} \rightarrow \mathbf{v}\right) n\left(\mathbf{r}^{\prime}, \mathbf{v}^{\prime}, t\right),
\end{array}
$$

E a equação de Boltzmann, reduz-se a uma equação de transporte linear, que no caso de nêutrons, o termo de transferência inclui a multiplicidade devido às colisões devido às fissões, ou seja

$$
\begin{gathered}
\frac{1}{v} \frac{\partial \Phi(\mathbf{r}, \boldsymbol{\Omega}, E, t)}{\partial t}=-\boldsymbol{\Omega} . \nabla \Phi-\Sigma_{t} \Phi+ \\
\int_{\Omega} d \boldsymbol{\Omega}^{\prime} \int_{0}^{\infty} d E^{\prime} \Sigma_{s}\left(\mathbf{r}, \boldsymbol{\Omega}^{\prime}, E^{\prime} \rightarrow \Omega, E ; t\right) \Phi\left(\mathbf{r}, \boldsymbol{\Omega}^{\prime}, E^{\prime}, t\right)+ \\
\frac{\chi(E)}{4 \pi} \int_{\Omega} d \boldsymbol{\Omega}^{\prime} \int_{0}^{\infty} d E^{\prime} v\left(E^{\prime}\right) \Sigma_{f}\left(\mathbf{r}, E^{\prime}\right) \Phi\left(\mathbf{r}, \boldsymbol{\Omega}^{\prime}, E^{\prime}, t\right)+S_{\text {ext }} .
\end{gathered}
$$

onde na equação de Boltzmann linear para nêutrons, ao invés da densidade de partículas introduz-se a grandeza fluxo, $\Phi=n v$, pela possibilidade do cálculo das taxas de reação, $\Sigma \Phi$ e a secção de choque macroscópica, ou probabilidade de interação por unidade de caminho, como $\Sigma=N \sigma$, com $N$ a densidade atômica do meio em que ocorre o transporte, e $\sigma$ a secção de choque microscópica, que em termos microscópicos descreve a interação dos nêutrons com os núcleos do meio, com os subscritos $s$ e $f$ para as reações de espalhamento e fissão (assumida isotrópica), e a secção de choque de transferência por espalhamento, o produto da densidade atômica do meio pela seção de choque diferencial de espalhamento $(\mathrm{d} \sigma / d E d \Omega)$, e o espaço de fase é a posição, direção e energia. No termo de fissão, $\chi(E)$ é o espectro de energias dos nêutrons de fissão e $v(E)$ é o numero de nêutrons emitidos por fissões induzidas por nêutrons com energia $E$. Esta equação é a base para a descrição da população de nêutrons num sistema (e.g. reatores nucleares), e é a base para o projeto dos reatores nucleares, também conhecida como física dos reatores. O fato a se destacar é que esta equação pode ser 
interpretada como um balanço de partículas no espaço de fases.

Outro fato a destacar é que de maneira análoga que a fluidodinâmica dos meios contínuos é uma conseqüência direta da mecânica estatística, a introdução da chamada Lei de Fick, que correlaciona a corrente de nêutrons com um gradiente de fluxo, i.e. $\quad \mathbf{J}=-D \nabla \Phi$, reduz a equação de transporte a um "modelo do continuo" para nêutrons, denominada teoria da difusão.
Desta forma, indiretamente, L. Boltzmann, é de certa forma o "pai" da física dos reatores nucleares, e mesmo na sua época sem o conhecimento das reações nucleares, e da mecânica quântica, será a sua equação a base para o projeto dos reatores nucleares atuais, e a nossa comunidade de física de reatores tem este brilhante cientista como o pilar de seu trabalho.

José Rubens Maiorino E-mail: maiorino@ipen.br

\section{Sobre os potenciais de condutores em movimento}

Publicamos em 2001 artigo nesta Revista [1], intitulado 'Esfera condutora em movimento: campo, potenciais e dúvidas'. As 'dúvidas' se referiam ao sentido físico do potencial escalar da esfera carregada em movimento e, principalmente, ao do potencial vetor que devemos atribuir à mesma. Com referência à Fig. 1, reproduzida daquele artigo, os principais pontos a recordar são os seguintes:

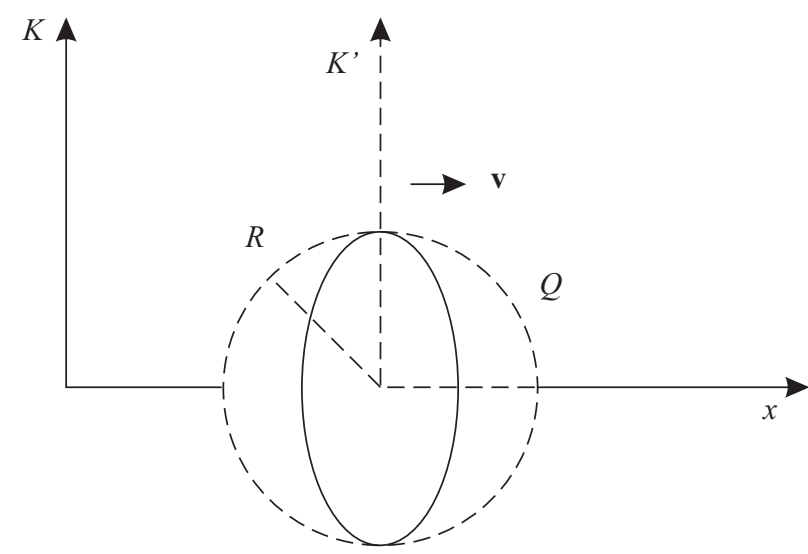

Figura 2 - Esfera no sistema $K^{\prime}$, em tracejado, vista do sistema $K$, traço cheio.

1) Para um observador no sistema $K$, a 'esfera' é um elipsóide achatado na direção de movimento e de revolução nas outras duas.

2) O potencial escalar do condutor em movimento, $\Phi$ em $K$, é maior do que o mesmo em $K^{\prime}, \Phi^{\prime}$, este igual a $Q / R$, pelo fator $\gamma=\left(1-v^{2} / c^{2}\right)^{-1 / 2}$, ou seja $\Phi=\gamma \Phi^{\prime}$. Os símbolos têm o sentido habitual, $Q$, carga, $R$, raio, $v$, velocidade da esfera e $c$, velocidade da luz.

3) Além do potencial escalar $\Phi$, devemos atribuir à 'esfera' condutora em movimento um potencial vetor, com uma componente exclusivamente na direção de movimento $A_{x}=v \Phi / c$

4) No sistema $K$, a normal ao condutor está na direção do 'campo' de Lorentz local, $\mathbf{E}+\mathbf{v} \times \mathbf{B} / c$.

5) O aumento do potencial escalar em $K$ deve ser atribuído ao fato de que nele os potenciais efetivos são os de Liénard-Wiechert, que, lembramos, não são simplesmente retardados pois incluem o fator de "paralaxe cinética', $(1-\mathbf{v} \cdot \hat{\mathbf{r}} / c)^{-1}$, sendo $\hat{r}$ o vetor unitário de $\mathbf{r}$ ao ponto considerado [2].

Gostaríamos de fazer aqui os seguintes esclarecimentos em relação ao trabalho anterior:

I) Os potenciais que o observador em $K$ atribui são sistêmicos, isto é, ao condutor em movimento como um todo. Notemos que o potencial escalar $\Phi$ é maior que $\Phi^{\prime}$, este medido em $K^{\prime}$, pelo fator $\gamma$, ou seja, na mesma proporção em que a massa relativística aumenta ao se passar de $K$ ' a $K$. Se adotamos o ponto de vista de que massa e energia são equivalentes, o aumento da energia potencial representa o aumento da 'energia elétrica' (ver ítem a seguir) do condutor em movimento.

II) Para nossa surpresa, se calcularmos as integrais dos quadrados dos campo elétrico e magnético no exterior do elipsóide, a grandeza obtida não parece guardar nenhuma relação direta com $\Phi$ [4]. Por esta razão, chamamos este, ou seu produto com $Q$, de 'energia elétrica', ligado ao conceito de potencial eletrostático ao qual os elétrons metálicos seriam sensíveis, imóveis ou móveis.

III) Tendo em conta o resultado mencionado no ítem 4) acima, concluimos que as transformações de Lorentz permitem encontrar a forma do condutor em movimento no qual a normal ao mesmo está na direção da força de Lorentz em cada ponto de sua superfície.

IV) A dúvida principal deixada no artigo se referia à interpretação a ser dada ao potencial vetor do condutor em movimento, igual a $v \Phi / c$ na direção de movimento. As considerações feitas no item I) sugerem que ele represente a quantidade de movimento associado à 
energia elétrica, necessariamente unida à energia numa entidade única 'energia-momento', como um quadrivetor da relatividade ou um multivetor 0-1 da álgebra geométrica, ou de Clifford.

V) Sabemos que se a esfera é supercondutora, seus pares bosônicos são sensíveis ao potencial vetor, de tal maneira que - na linguagem de Feynman [3] - o $p$ momento, igual à soma do $m v$-momento e ao produto da carga pelo potencial vetor (ou momento elétrico, na nossa), é conservado. Se imaginamos que ela, inicialmente parada, recebe impulsivamente a velocidade $v$ no sistema $K$, temos que o $p$-momento, inicialmente nulo, assim permanece porque para cada elétron no interior do condutor, vale a relação $m v+q A_{x}=0$ (pois $q$, carga do elétron, é negativa) de acordo com a argumentação desenvolvida em IV.

G.F. Leal Ferreira

DFCM, IF - São Carlos, E-mail: guilherm@if.sc.usp.br

\section{Referências}

[1] G.F. Leal Ferrreira, Rev. Bras. Ens. Fis. 23, 141 (2001).

[2] R.P. Feynman, R.B. Leighton e M. Sands, The Feynman Lectures on Physics, $v$.2 (Addison Wesley, Reading, 1965).

[3] V. 3 da Ref. [2].

[4] Obtivemos para $(1 / 8 \pi) \int_{0}^{\pi} 2 \pi \operatorname{sen} \theta d \theta \int_{r(\theta)}^{\infty}\left(E^{2}+\right.$ $\left.B^{2}\right) r^{2} d r$ o resultado $Q^{2}\left(3-v^{2} / c^{2}\right) /\left(6 R \sqrt{1-v^{2} / c^{2}}\right)$. 